\title{
Baselines representing blood glucose clearance improve in vitro prediction of the glycaemic impact of customarily consumed food quantities
}

\author{
John A. Monro ${ }^{1}$, Suman Mishra ${ }^{1}$ and Bernard Venn ${ }^{2}$ \\ ${ }^{1}$ New Zealand Institute for Plant and Food Research Ltd, Private Bag 11 600, Palmerston North, New Zealand \\ ${ }^{2}$ Department of Human Nutrition, University of Otago, Dunedin, New Zealand
}

(Received 29 January 2009 - Revised 16 June 2009 - Accepted 23 July 2009 - First published online 24 November 2009)

Glycaemic responses to foods reflect the balance between glucose loading into, and its clearance from, the blood. Current in vitro methods for glycaemic analysis do not take into account the key role of glucose disposal. The present study aimed to develop a food intake-sensitive method for measuring the glycaemic impact of food quantities usually consumed, as the difference between release of glucose equivalents (GGE) from food during in vitro digestion and a corresponding estimate of clearance of them from the blood. Five foods - white bread, fruit bread, muesli bar, mashed potato and chickpeas - were consumed on three occasions by twenty volunteers to provide blood glucose response (BGR) curves. GGE release during in vitro digestion of the foods was also plotted. Glucose disposal rates estimated from downward slopes of the BGR curves allowed GGE dose-dependent cumulative glucose disposal to be calculated. By subtracting cumulative glucose disposal from cumulative in vitro GGE release, accuracy in predicting the in vivo glycaemic effect from in vitro GGE values was greatly improved $\mathrm{GGE}_{\text {in vivo }}=0.99 \mathrm{GGE}_{\text {in vitro }}+0.75\left(R^{2} 0.88\right)$. Furthermore, the difference between the curves of cumulative GGE release and disposal closely mimicked in vivo incremental BGR curves. We conclude that valid measurement of the glycaemic impact of foods may be obtained in vitro, and expressed as grams of glucose equivalents per food quantity, by taking account not only of GGE release from food during in vitro digestion, but also of blood glucose clearance in response to the food quantity.

Carbohydrate: Glycaemic impact: Glucose disposal: In vitro digestion

For practical consumer and dietetic use in glycaemia management, food values are required which will '... communicate glycaemic response in grams per serving of food'(1), and which should be measured using '... validated in vitro methodology that accurately mimics in vivo behaviour' ${ }^{(2)}$.

Current in vitro digestion methods for predicting relative glycaemic responses to foods measure carbohydrate that is rapidly available (for example, Englyst et al. ${ }^{(3)}$, Brighenti et $\left.a l .{ }^{(4)}\right)$, or the area under the glucose release curve relative to a reference ${ }^{(5)}$. However, they cannot provide accurate or robust predictions of relative responses to whole foods varying in quantity, or accurately mimic the in vivo glycaemic response, because they do not allow for the effects of homeostasis. They do not take into account the fact that glycaemic responses to foods are a net effect of the balance between blood glucose loading and blood glucose disposal (GD), which depends dynamically on the rates of both. Not allowing for blood GD, and its dependence on the amount and rate of blood glucose loading, may lead to large inaccuracies when using glycaemic impact values from experimental portions to gauge the effect of the very different and varying food quantities that are consumed in a community setting. For instance, customarily consumed food portions often have a much lower glycaemic impact than would be expected from a glycaemic load value calculated from the glycaemic index ${ }^{(6)}$, because glycaemic index is based on a $50 \mathrm{~g}$ glucose reference, which is much greater than the glucose equivalents that would be consumed in most servings of foods. With the need for in vitro methods that predict the relative glycaemic effect of realistic intakes of food ${ }^{(7)}$, methods that take account of the effects of homeostatic responses to dose are required.

A recent well-replicated ( $n$ 20) clinical study of the glycaemic effects of five foods fed on three occasions, and glucose fed on four occasions, provided glucose response curves and clinical glycaemic glucose equivalent (GGE) values for the foods ${ }^{(8)}$. In vitro digestion of the same foods has given us curves of cumulative carbohydrate release. With detailed in vivo and in vitro datasets for the same foods we have an opportunity to measure improvements in the predictive validity of in vitro measures when glucose homeostasis is allowed for.

In the present paper we test the hypothesis that the difference between the cumulative release of GGE during in vitro digestion, adjusted for food intake, and the estimated cumulative disposal of them that would occur in the body will provide an accurate and intake-sensitive prediction of the relative glycaemic response in vivo. The aim is to develop in vitro methods that are flexible enough to accurately provide glycaemic impact values for the varying quantities of foods, of different carbohydrate composition, that are customarily consumed by humans. 


\section{Materials and methods}

Food samples

The five test foods were all purchased from a supermarket and were white bread (Tip Top, Family Fresh Super Soft), fruit bread (Burgen), muesli bar (Mother Earth, sultana, oat and honey), instant mashed potatoes and canned chickpeas.

\section{Digestive analysis in vitro}

The food samples were provided to subjects who each took a mouthful of the size they would habitually consume, chewed until the urge to swallow, and then expectorated the mouthful into a beaker. For each food, the expectorated samples of all participants were immediately mixed in equal volumes and duplicate subsamples of the pooled material were quickly weighed into digestion pots. The chewed sample weights used were: white bread, $2.5 \mathrm{~g}$; fruit bread; $2.5 \mathrm{~g}$; muesli bar; $2.5 \mathrm{~g}$; mashed potato, $10 \mathrm{~g}$; chickpeas, $5.0 \mathrm{~g}$ (all $\pm 0.2 \mathrm{~g}$ ).

Food samples were digested in $70 \mathrm{ml}$ specimen pots inserted to their full depth in a fifteen-place aluminium heating block placed on a fifteen-place magnetic stirrer and covered with an insulating sheet. The digestion consisted of a simulated gastric digestion followed by an ileal digestion, with timed sampling during the small-intestinal phase. Water to $30 \mathrm{ml}$ and $0.8 \mathrm{ml}$ of $1 \mathrm{M}-\mathrm{HCl}$ were added to the sample to attain $\mathrm{pH} 2.5( \pm 0.2)$, with $\mathrm{pH}$ adjustment if necessary. Then $1 \mathrm{ml}$ of $10 \%$ pepsin dissolved in $0.05 \mathrm{M}-\mathrm{HCl}$ was added, and the mixture stirred slowly and intermittently $(15 \mathrm{~s}$ on, $15 \mathrm{~s}$ off, at $130 \mathrm{rpm}$ ) for $30 \mathrm{~min}$ at $37^{\circ} \mathrm{C}$ to accomplish gastric digestion. The small-intestinal phase was initiated by neutralising the gastric $\mathrm{HCl}$ with $2 \mathrm{ml}$ of $1 \mathrm{M}-\mathrm{NaHCO}_{3}$ and $5 \mathrm{ml}$ of $0 \cdot 1 \mathrm{M}-$ sodium maleate buffer ( $\mathrm{pH} 6)-0.2 \%$ sodium azide- $1 \mathrm{mM}$ $\mathrm{CaCl}_{2}$. Starch digestion was commenced by adding, in quick succession, $0.1 \mathrm{ml}$ amyloglucosidase (catalogue no. E-AMGDF; Megazyme International, Bray, Co. Wicklow, Republic of Ireland) and $5 \mathrm{ml}$ of $2.5 \%$ pancreatin (Sigma P-7545; Sigma, St Louis, MO, USA) in 0.1 M-maleate buffer ( $\mathrm{pH} \mathrm{6)}$, and the pots were immediately made to the $55 \mathrm{ml}$ mark with distilled water. Duplicate $1.0 \mathrm{ml}$ samples were each removed to $4 \mathrm{ml}$ ethanol, before $(\mathrm{T}=0)$, and at 10,20 , $30,40,60$ and $120 \mathrm{~min}$ after adding the amyloglucosidasepancreatin. After the $120 \mathrm{~min}$ sampling the digests were homogenised using an Omni GLH-220 homogeniser $(20 \times 195 \mathrm{~mm}$ saw tooth probe, setting 3, $15 \mathrm{~s}$; Omni International, Warrenton, VA, USA) to convert them into slurries. A further $0.1 \mathrm{ml}$ of amyloglucosidase was added and the incubation continued for $2 \mathrm{~h}$ with further $1 \mathrm{ml}$ samples removed to $4 \mathrm{ml}$ ethanol at 180 and at $240 \mathrm{~min}$ from the start of digestion to determine total available carbohydrate. All digesta samples were mixed thoroughly upon adding to the ethanol, and after at least $30 \mathrm{~min}$ the tubes were centrifuged for $10 \mathrm{~min}$ at $1000 \mathrm{~g}$ at $20^{\circ} \mathrm{C}$ (Centrifuge Omnifuge $2.0 \mathrm{RS}$; Heraeus Sepatech, Osterade, Germany) to clarify the $80 \%$ ethanol supernatant fraction before analysis of sugars.

\section{Measuring glucose equivalents released during in vitro digestion}

Sugars released during digestion were measured after an invertase + amyloglucosidase secondary digestion as glucose equivalents by a small-scale modification of the dinitrosalicylic acid colorimetric method ${ }^{(9)}$, using glucose references. A $0.05 \mathrm{ml}$ aliquot of ethanolic sample from the in vitro digestion above, or glucose standard $(1 \mathrm{mg} / \mathrm{ml}$ glucose $)$, was added to $0.25 \mathrm{ml}$ of $0.2 \mathrm{M}$-acetate buffer ( $\mathrm{pH} \mathrm{5.2)} \mathrm{con-}$ taining $1 \%$ of amyloglucosidase (catalogue no. E-AMGDF; Megazyme International) $+1 \%$ of invertase concentrate (catalogue no. 390203D; BDH, Poole, Dorset, UK) and incubated at $37^{\circ} \mathrm{C}$ for $10 \mathrm{~min}$ to complete depolymerisation to monosaccharides. Reducing sugars were then measured by adding $0.75 \mathrm{ml}$ dinitrosalicylic mixture (containing a $1: 1: 5$ mixture of $0.5 \mathrm{mg} / \mathrm{ml}$ glucose $-4 \mathrm{M}-\mathrm{NaOH}-\mathrm{DNS}$ reagent) and heated for $15 \mathrm{~min}$ at $95-100^{\circ} \mathrm{C}$. The tubes were cooled, $4.0 \mathrm{ml}$ water added, mixed, and absorbances read at $530 \mathrm{~nm}$ (Jenway 6100 Spectrophotometer; Jenway Ltd, Dunmow, Essex, UK). The DNS reagent consisted of $10 \mathrm{~g}$ of 3,5-dinitrosalicylic acid dissolved in 1 litre of solution containing $300 \mathrm{~g} \mathrm{Na}-\mathrm{K}$ tartarate and $16 \mathrm{~g} \mathrm{NaOH}$. The fructose content of the digests was determined by the thiobarbituric acid method ${ }^{(10)}$.

The weight of food in the chewed samples was determined by measuring available carbohydrate in the exact amount of food in the non-chewed homogenised samples. The results were then plotted as glucose equivalent release $(\mathrm{mg})$ per $\mathrm{g}$ of unchewed sample $v$. time (Fig. 1).

Converting in vitro glucose equivalents to glycaemic glucose equivalents

In vitro GGE values were obtained by removing the fructose component of glucose equivalent (GE) (GE - fructose), and then adding back the amount of glucose that would be equivalent in its glycaemic effect to the removed fructose $\left(0 \cdot 2\right.$ fructose, as the glycaemic index of fructose is $\left.20 \%{ }^{(11)}\right)$ :

$$
\mathrm{GGE}(\mathrm{g})=(\mathrm{GE}-\text { fructose })+0 \cdot 2 \text { fructose } .
$$

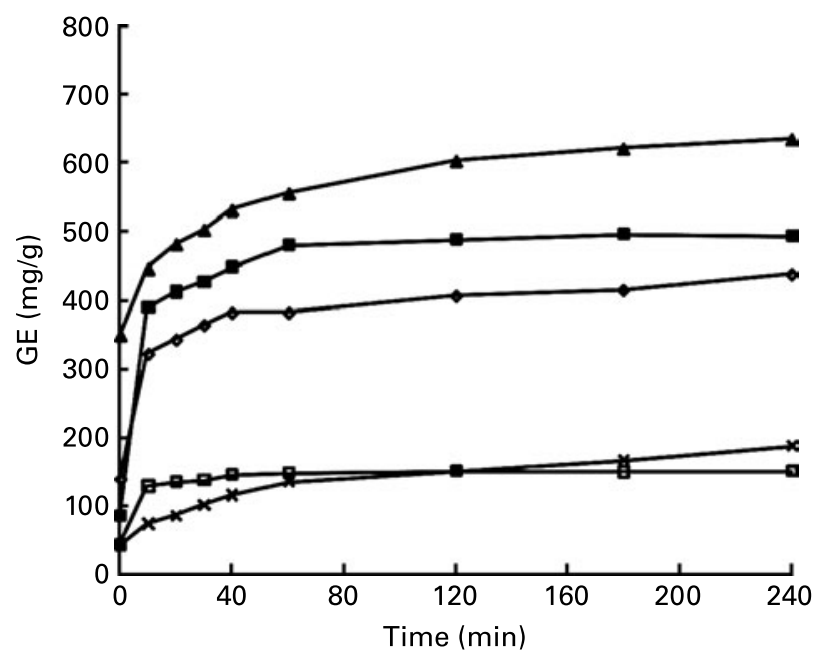

Fig. 1. Release of carbohydrate during in vitro digestion measured as glucose equivalents (GE) and expressed per $\mathrm{g}$ of unchewed food. Each point is the mean of two determinations. Precision based on mean SD for all determinations was $\pm 8.8 \mathrm{GE}$. Mean CV was $3.2 \%$. $\left(-\diamond_{-}\right)$, Fruit bread; $(-\square-)$, potato; (-口-), white bread; $(-\times-)$, chickpeas; $(-\boldsymbol{\Delta}-)$, muesli bar. 


\section{Blood glucose responses}

Blood glucose response curves were generated from background data to an earlier study for which ethics approval, subjects and methods have been described in detail ${ }^{(8)}$. The present study was conducted according to the guidelines laid down in the Declaration of Helsinki and all procedures involving human subjects were approved by the Human Ethics Committee of the University of Otago. Written informed consent was obtained from all subjects. Foods were consumed at a serving size of $100 \mathrm{~g}$ and at an intake that would deliver $50 \mathrm{~g}$ available carbohydrate, except for chickpeas where enough was consumed to provide $25 \mathrm{~g}$ available carbohydrate (Table 1). Standard procedures were used, with capillary blood sampling and calculation of the incremental area under the blood glucose response curve using the triangle/trapezoid summation method described by the FAO/WHO ${ }^{(12)}$. For each intake of food and glucose reference doses the blood glucose responses for all individuals at each blood sampling time were averaged and plotted against time, to give an average blood glucose response curve for each intake of each food and reference (Fig. 2). Smoothed curves were fitted to the data points using the Excel chart-plotting program and zero response at time zero was assumed. Errors associated with the blood glucose response measurements have been described in Venn et al. ${ }^{(8)}$ and Table 1.

\section{Establishing glucose disposal baselines}

Rates of GD were estimated from the blood glucose response curves at each food intake by triangulation, in which the area under the linear portion of the post-peak decline in blood

Table 1. Foods and food quantities used in clinical determination of glycaemic glucose equivalents (GGE) and in calculating corresponding glucose equivalents and GGE from in vitro digestion of chewed samples

(Mean values and standard deviations)

\begin{tabular}{lccr}
\hline & & \multicolumn{2}{c}{$\mathrm{GGE}_{\text {in vivo }}{ }^{*}(\mathrm{~g})$} \\
\cline { 3 - 4 } Quantity & Food intake $(\mathrm{g})$ & Mean & $\mathrm{SD}$ \\
\hline White bread & & & \\
$\quad$ One serving & 37 & 12 & $5 \cdot 9$ \\
$100 \mathrm{~g}$ & 100 & 28 & $11 \cdot 7$ \\
$50 \mathrm{~g}$ CHOAVL & 118 & 41 & $9 \cdot 5$ \\
Fruit loaf & & & \\
One serving & 41 & 9 & $6 \cdot 8$ \\
$100 \mathrm{~g}$ & 100 & $37 \cdot 5$ & $6 \cdot 9$ \\
$50 \mathrm{~g}$ CHOAVL & 132 & & $16 \cdot 3$ \\
Muesli bar & & $13 \cdot 9$ & \\
One bar & 50 & 23 & $5 \cdot 9$ \\
Two bars & 100 & $30 \cdot 3$ & $13 \cdot 8$ \\
Three bars & 150 & & $13 \cdot 2$ \\
Mashed potato & & $10 \cdot 8$ & \\
100 g & 100 & $15 \cdot 3$ & $6 \cdot 1$ \\
One serving & 140 & $42 \cdot 6$ & $6 \cdot 7$ \\
$50 \mathrm{~g}$ CHOAVL & 472 & $3 \cdot 9$ & $15 \cdot 1$ \\
Chickpeas & & $4 \cdot 9$ & $4 \cdot 3$ \\
One serving & 70 & 8 & $4 \cdot 5$ \\
$100 \mathrm{~g}$ & 100 & & $6 \cdot 7$ \\
$25 \mathrm{~g}$ CHOAVL & 136 & \\
\hline
\end{tabular}

CHOAVL, available carbohydrate.

* Venn et al. ${ }^{(8)}$.

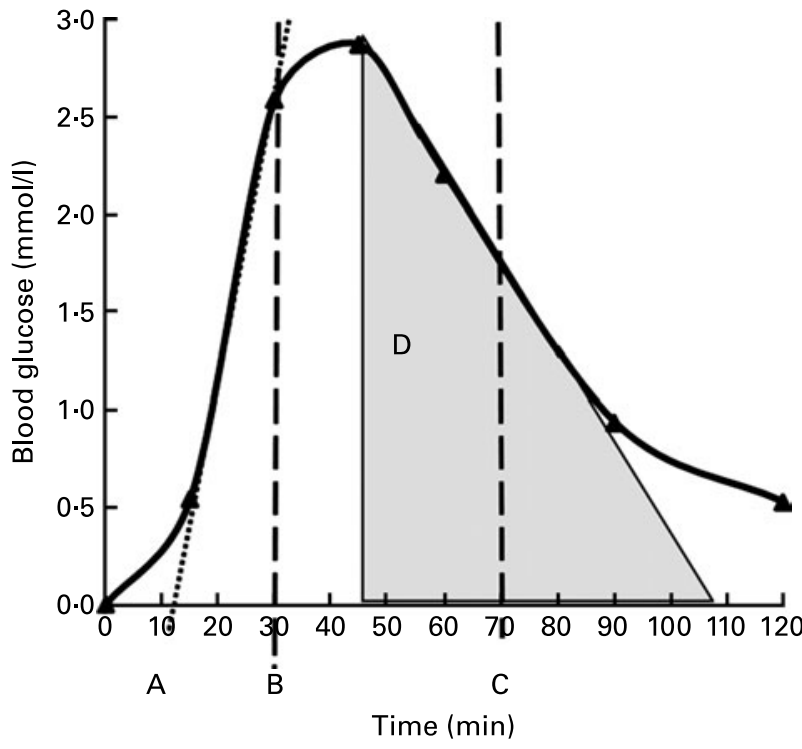

Fig. 2. Blood glucose responses for all individuals following the consumption of white bread at each blood sampling time averaged and plotted against time. Line $A(\cdots . . .$.$) : the delay in glucose loading was approximately 10 \mathrm{~min}$. Line B (-.--): the maximum net glucose loading rate was achieved by about $20 \mathrm{~min}$. Line C (--.--): an appropriate glycaemic glucose equivalent (GGE) value on which to base glucose disposal was that which had been released at $60 \mathrm{~min}$. Area $\mathrm{D}(\square)$ : the glucose disposal rate was calculated as GGE loss per min from the area under the linear post-peak slope $=$ (total GGE $\times$ triangle area/total area)/time using GGE in vivo values (Table 1). The disposal rates were then adjusted for non-linearity in the glucose standard (Fig. 3) before using them to generate lines of cumulative glucose disposal.

glucose was measured. As the GGE quantity represented by the area under the whole curve had already been determined $^{(8)}$, the GGE disposal rate could be calculated as:

$$
\operatorname{GD} \text { rate }(\mathrm{GGE} / \mathrm{min})=\left(\left(\mathrm{A}_{\mathrm{D}} / \mathrm{A}_{\text {total }}\right) \times \mathrm{GGE}_{\text {total }}\right) / \mathrm{T}_{\mathrm{h}},
$$

where $A_{D}$ is the area under the hypotenuse, $A_{\text {total }}$ is the total area under the blood glucose response curve, $\mathrm{GGE}_{\text {total }}$ is the GGE value for the total area and $\mathrm{T}_{\mathrm{h}}$ is the time ( $x$-axis) under the hypotenuse (Fig. 2).

This procedure gave a set of GGE disposal rates which were estimates because they represent change in the net balance between blood glucose loading and clearance, and are measured over the portion of the blood glucose response curve covering most, but not all, of the decline in blood glucose concentrations.

Because the clinically determined GGE values had used glucose references that were also subject to homeostasis the values were adjusted for the quadratic decline in the glucose reference response (Fig. 3). The adjusted GD rates were then plotted against their corresponding GGE intakes to provide a graph and equation for GD rate as a function of GGE intake (Fig. 4). The equation was used to identify GD rates with which to calculate cumulative GD baselines as GD rate $\times$ time for all fifteen food intakes.

\section{Modelling glycaemic response as the balance between glycaemic glucose equivalent loading and glucose disposal}

GGE released per $\mathrm{g}$ of food after $60 \mathrm{~min}$ in vitro digestion was multiplied by the food intake, and entered into the 


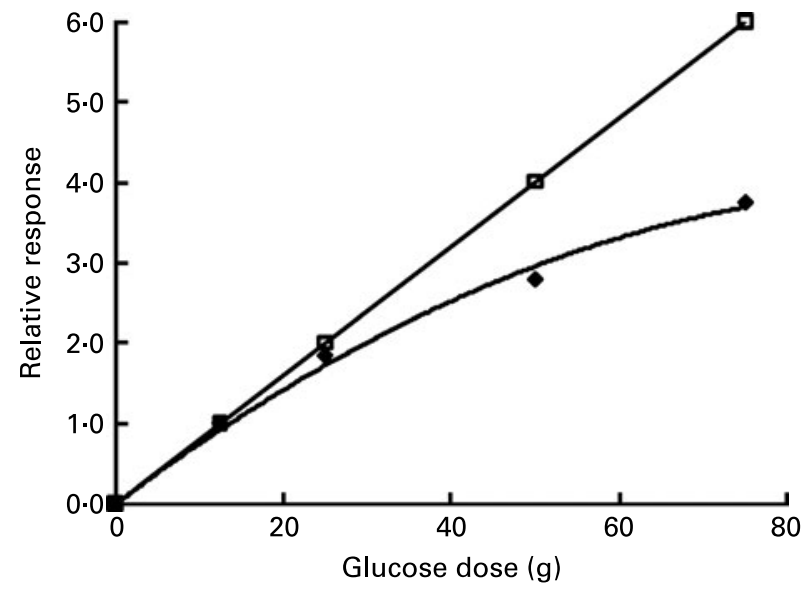

Fig. 3. Quadratic increase in actual blood glucose response with increasing glucose doses $(-\downarrow-)$, compared with a linear extrapolation from the origin through the response to $12.5 \mathrm{~g}$ glucose (assigned a value of 1.0$)(-\square-)$. The divergence between the curves is used as a measure of the effect of homeostasis on the blood glucose response.

equation of disposal rate $v$. GGE intake (GD rate (in GGE/ $\min )=0.0135 \times$ GGE intake (in $\mathrm{g})+0 \cdot 0232$; Fig. 4) to obtain predicted GGE disposal rates (GGE/min) for the various food intakes (Table 1). From these predicted GD rates, baselines of cumulative GGE removal (GD rate $\times$ time) could be plotted within the graphs of cumulative GGE release (Fig. 5).

GD rates in response to GGE released after $60 \mathrm{~min}$ in vitro digestion were used to calculate cumulative GGE disposal (Fig. 5), because the linear declines in blood glucose from which GD rates were calculated were completed about $60 \mathrm{~min}$ after the initiation of the blood glucose response (10 min delay plus $60 \mathrm{~min}$ ) (Figs. 2 and 6(b)). Most of the GGE release in vitro at $60 \mathrm{~min}$ had occurred by

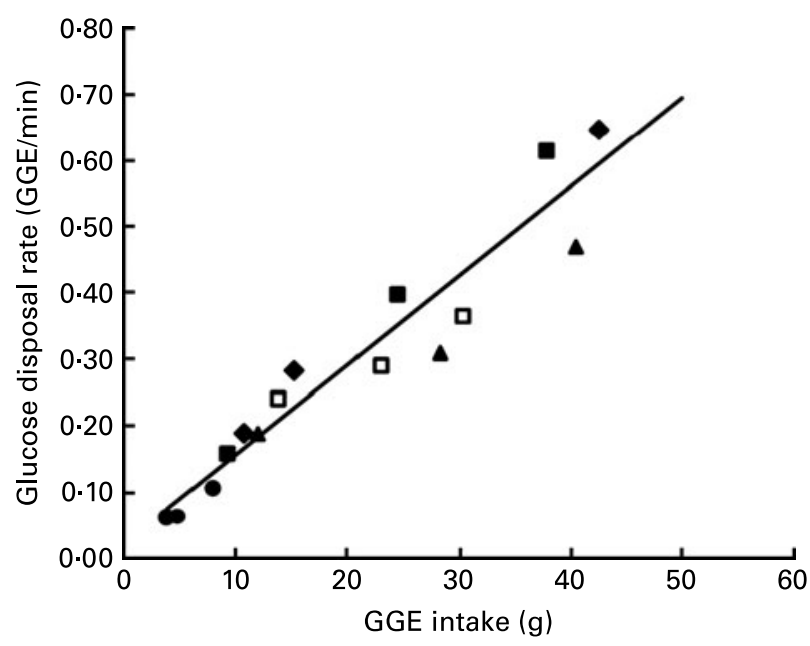

Fig. 4. Glucose disposal rates measured by triangulation of the negative post-peak blood glucose response slope (Fig. 2) as a function of glycaemic glucose equivalents (GGE) $\left(y=0.0135 x+0.0232 ; R^{2} 0.93\right)$, after correcting for non-linearity using the ratios of the linear to quadratic responses to glucose (Fig. 3). ( $\square$ ), Muesli bar; $(\bullet)$, potato; $(\boldsymbol{\Delta})$, white bread; $(\bullet)$, chickpeas; $(\square)$, fruit loaf; (-), all foods.

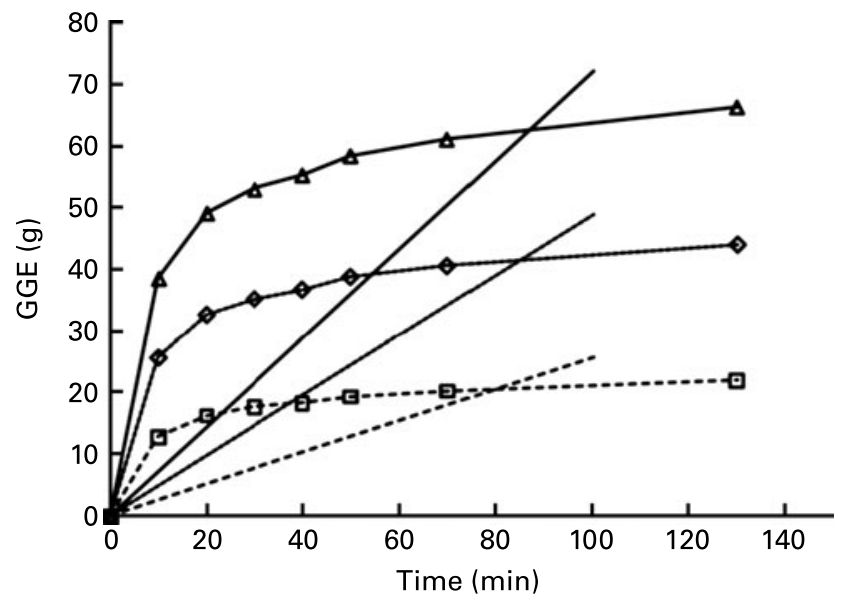

Fig. 5. Release of glycaemic glucose equivalents (GGE) calculated from glucose equivalents for three intakes of muesli bar (lines with markers: $-\Delta-$, $150 \mathrm{~g} ; \cdots \diamond \cdots, 100 \mathrm{~g} ;--\square--, 50 \mathrm{~g})$, and straight lines $(y=$ glucose disposal (GD) rate $\times$ time: - , GD $150 \mathrm{~g} ; \cdots .$. , GD $100 \mathrm{~g}$; -..-, GD $50 \mathrm{~g}$ ) for cumulative GD. GD rate was calculated by substituting in vitro GGE release at $60 \mathrm{~min}$ for the given food intakes into the equation relating GGE intake to GD rate $(\mathrm{GD}$ rate $=0.0135 \mathrm{GGE}+0.0232 ;$ Fig. 4$)$.

20 min digestion, so the same GD baseline could be used for a two-point net GGE determination based on rapidly available carbohydrate $(20 \mathrm{~min})$ minus cumulative $\mathrm{GD}$ at $20 \mathrm{~min}$, but using the GD rate for a $60 \mathrm{~min}$ GGE intake. From the graphs containing a curve of GGE release and a line for GD for each intake of each food (Fig. 5), values for net glycaemic loading were obtained at each time point as the difference between cumulative GGE release and cumulative GD at the time (Table 2). The values for net glycaemic loading were then divided by the same adjustment factors as had been used to compensate for the nonlinear glucose reference curve (for example, Table 2) and these readjusted net glycaemic loading values were plotted against time, to provide simulated blood glucose response curves (Fig. 6(a)) that represented the adjusted areas between the curves of GGE release and GD, and which could be compared with the unadjusted blood glucose responses (Fig. 6(b)).

Measuring net glycaemic glucose equivalent content using in vitro area between the glycaemic glucose equivalent loading and disposal curves

The areas between the lines of cumulative in vitro GGE release and GD (Fig. 6) were measured using the triangle/trapezoid summation method described by the $\mathrm{FAO} / \mathrm{WHO}^{(12)}$ (Table 3). One slice of white bread, which contained $12.0 \mathrm{~g}$ GGE in an area of 343.6 GGE $\times$ min, was used as a reference of known area per GGE. The areas (A) between the in vitro GGE release and GD lines calculated for the other food quantities were thus converted to GGE values using the relationship $\mathrm{GGE}=\mathrm{A} \times 12 / 343.6(\mathrm{~g})$. The values obtained were then correlated with the clinically determined GGE values for the foods (Fig. 7) and a comparison of methods was made with a Bland-Altman analysis of the in vitro and in vivo datasets. 

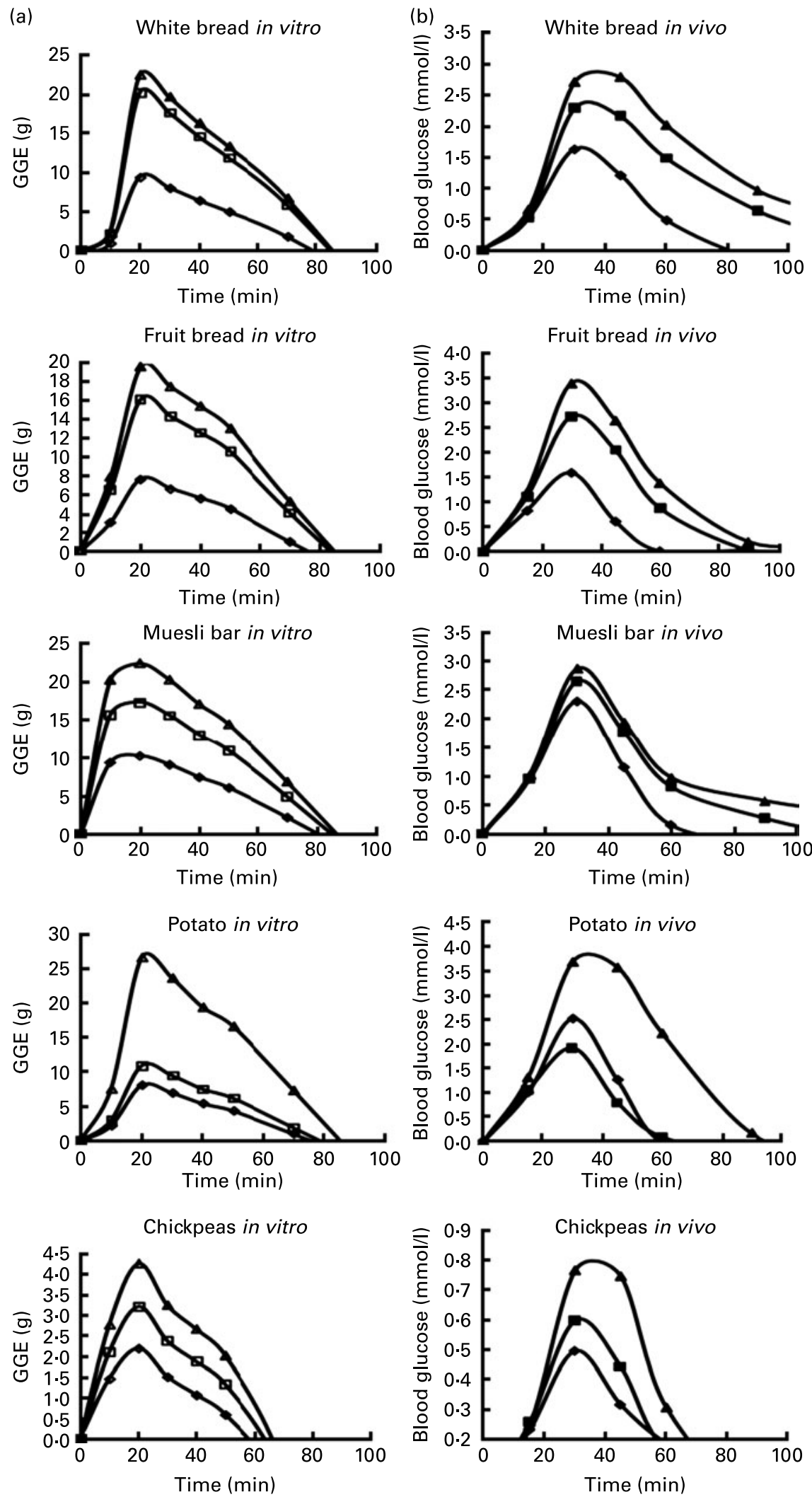

Fig. 6. Profiles of net glycaemic loading in vitro measured as the difference between cumulative glycaemic glucose equivalent (GGE) release in vitro (a) and cumulative theoretical glucose disposal, compared with in vivo blood glucose responses (b). White bread in vitro: $(-\diamond-)$, $37 \mathrm{~g} ;(-\square-)$, $100 \mathrm{~g} ;(-\triangle-), 118 \mathrm{~g}$. White

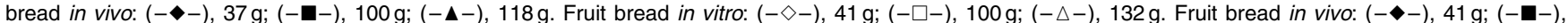
$100 \mathrm{~g} ;(-\boldsymbol{\Delta}-)$, $132 \mathrm{~g}$. Muesli bar in vitro: $(-\diamond-), 50 \mathrm{~g} ;(-\square-), 100 \mathrm{~g} ;(-\Delta-), 150 \mathrm{~g}$. Muesli bar in vivo: $(-\diamond-), 50 \mathrm{~g} ;(-\mathbf{\square}-), 100 \mathrm{~g} ;(-\mathbf{\Delta}-), 150 \mathrm{~g}$. Potato in vitro:

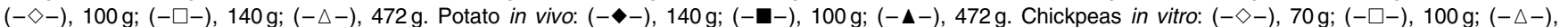
$136 \mathrm{~g}$. Chickpeas in vivo: $(--), 70 \mathrm{~g} ;(-\mathbf{-}-), 100 \mathrm{~g} ;(-\boldsymbol{\Delta}-), 136 \mathrm{~g}$. 
Table 2. Example of spreadsheet for calculating area between the in vitro glycaemic glucose equivalents (GGE) release and glucose-disposal curves: muesli bar

\begin{tabular}{|c|c|c|c|c|c|c|c|c|c|c|c|c|c|c|c|}
\hline Intake (g) & 50 & & & & & 100 & & & & & 150 & & & & \\
\hline $\begin{array}{l}\text { Glucose equivalents to GGE } \\
\text { conversion }\end{array}$ & 0.733 & & & & & 0.733 & & & & & 0.733 & & & & \\
\hline Glucose disposal (GGE/min) & & 0.256 & & & & & 0.487 & & & & & 0.719 & & & \\
\hline Linearising factor & & & & 1.09 & & & & & 1.32 & & & & & 1.54 & \\
\hline Time (min) & $\begin{array}{c}\text { GGE } \\
(\mathrm{g})^{\star}\end{array}$ & BL† & Diff & Diff adj§ & iAUC & $\begin{array}{l}\text { GGE } \\
(\mathrm{g})^{*}\end{array}$ & $\mathrm{BL} \dagger$ & Diff $\neq$ & Diff adj§ & iAUC & $\begin{array}{l}\text { GGE } \\
(\mathrm{g})^{*}\end{array}$ & $\mathrm{BL} \dagger$ & Diff & Diff adj§ & iAUC \\
\hline 0 & 0 & 0 & 0 & 0 & & 0 & 0 & 0 & 0 & & 0 & 0 & 0 & 0 & \\
\hline 10 & $12 \cdot 8$ & $2 \cdot 56$ & $10 \cdot 3$ & 9.4 & $47 \cdot 0$ & $25 \cdot 6$ & 4.87 & $20 \cdot 8$ & $15 \cdot 7$ & 78.6 & 38.4 & $7 \cdot 19$ & 31.3 & $20 \cdot 3$ & 101.5 \\
\hline 20 & $16 \cdot 3$ & $5 \cdot 12$ & 11.2 & $10 \cdot 3$ & 98.5 & $32 \cdot 6$ & 9.74 & $22 \cdot 9$ & $17 \cdot 3$ & $165 \cdot 4$ & $49 \cdot 0$ & 14.4 & 34.6 & 22.5 & $213 \cdot 8$ \\
\hline 30 & $17 \cdot 6$ & $7 \cdot 67$ & 9.9 & $9 \cdot 1$ & $97 \cdot 0$ & $35 \cdot 2$ & 14.62 & $20 \cdot 6$ & $15 \cdot 6$ & $164 \cdot 8$ & $52 \cdot 8$ & $21 \cdot 6$ & $31 \cdot 3$ & $20 \cdot 3$ & $213 \cdot 9$ \\
\hline 40 & $18 \cdot 4$ & $10 \cdot 23$ & 8.1 & 7.5 & $82 \cdot 9$ & $36 \cdot 7$ & $19 \cdot 49$ & $17 \cdot 3$ & $13 \cdot 1$ & 143.4 & $55 \cdot 1$ & $28 \cdot 8$ & $26 \cdot 4$ & $17 \cdot 1$ & $187 \cdot 2$ \\
\hline 50 & $19 \cdot 4$ & $12 \cdot 79$ & 6.6 & $6 \cdot 1$ & $67 \cdot 7$ & 38.8 & 24.36 & 14.5 & $10 \cdot 9$ & $120 \cdot 1$ & $58 \cdot 2$ & 35.9 & $22 \cdot 3$ & 14.5 & $158 \cdot 0$ \\
\hline 70 & $20 \cdot 3$ & 17.91 & $2 \cdot 4$ & $2 \cdot 2$ & 82.5 & $40 \cdot 6$ & $34 \cdot 11$ & 6.5 & 4.9 & 158.5 & 60.9 & $50 \cdot 3$ & $10 \cdot 6$ & 6.9 & $213 \cdot 3$ \\
\hline 130 & 22.0 & 33.26 & -11.2 & $-10 \cdot 3$ & $5 \cdot 2$ & 44.0 & 63.34 & $-19 \cdot 3$ & -14.6 & 7.5 & $66 \cdot 1$ & 93.4 & $-27 \cdot 4$ & -17.8 & 8.4 \\
\hline 190 & $22 \cdot 7$ & 48.61 & $-25 \cdot 9$ & $-23 \cdot 8$ & & 45.4 & 92.58 & $-47 \cdot 2$ & $-35 \cdot 8$ & & $68 \cdot 1$ & 136.6 & -68.5 & -44.5 & \\
\hline \multirow[t]{2}{*}{250} & 23.2 & 63.96 & $-40 \cdot 8$ & -37.4 & & $46 \cdot 4$ & $121 \cdot 81$ & -75.4 & $-57 \cdot 1$ & & $69 \cdot 6$ & 179.7 & -110.1 & -71.5 & \\
\hline & & & & Total\| & 481 & & & & Total\| & 838 & & & & Total\| & 1099 \\
\hline
\end{tabular}

${ }^{*}$ GGE $=$ in vitro glucose equivalents/g (Fig. 1) released at each time adjusted for glycaemic potency of the contributing sugars (0.733) and multiplied by food intake. $\dagger \mathrm{BL}=$ glucose disposal baseline $=$ time $(\mathrm{min}) \times$ glucose disposal factor determined from GGE release at $60 \mathrm{~min}$ using the equation in Fig. 4.

$\S$ Diff adj $=$ Diff after non-linearity correction based on the ratio of the linear to quadratic response as a function of GGE (Fig. 3).

$\|$ SiAUC $=$ sum of the areas under the Diff adj $\times$ time curve between each measurement point (Fig. 5), using the triangle/trapezoid method ${ }^{(12)}$ 
Table 3. Key measurement points from in vitro digestive analysis, and effects of converting glucose equivalents (GE) to glycaemic glucose equivalents (GGE)

(Mean values and standard deviations)

\begin{tabular}{|c|c|c|c|c|c|c|c|c|c|c|}
\hline \multirow[t]{2}{*}{ Food... } & \multicolumn{2}{|c|}{ White bread } & \multicolumn{2}{|c|}{ Fruit loaf } & \multicolumn{2}{|c|}{ Muesli bar } & \multicolumn{2}{|c|}{ Potato } & \multicolumn{2}{|c|}{ Chickpeas } \\
\hline & Mean & SD & Mean & SD & Mean & SD & Mean & SD & Mean & SD \\
\hline \multicolumn{11}{|l|}{ GE release $(\mathrm{g})$} \\
\hline $20 \min$ & $412 \cdot 6$ & $17 \cdot 7$ & 343.5 & $9 \cdot 3$ & $480 \cdot 6$ & 3.4 & $135 \cdot 5$ & $2 \cdot 8$ & $85 \cdot 8$ & $6 \cdot 2$ \\
\hline $60 \mathrm{~min}$ & 479.4 & $37 \cdot 3$ & 382.5 & $6 \cdot 3$ & 553.6 & $6 \cdot 1$ & 147.5 & 3.4 & $135 \cdot 1$ & 4.7 \\
\hline $240 \min$ & $492 \cdot 9$ & 9.4 & 438.4 & 3.5 & $632 \cdot 7$ & 8.9 & $150 \cdot 0$ & 1.5 & $186 \cdot 5$ & $2 \cdot 2$ \\
\hline GGE:GE ratio* & 1.00 & & 0.86 & & 0.73 & & 0.92 & & 1.00 & \\
\hline \multicolumn{11}{|l|}{ GGE release† $(\mathrm{g})$} \\
\hline $20 \mathrm{~min}$ & 411.4 & $17 \cdot 7$ & $295 \cdot 7$ & $8 \cdot 0$ & $352 \cdot 3$ & $2 \cdot 5$ & 124.4 & $2 \cdot 6$ & $85 \cdot 8$ & $6 \cdot 2$ \\
\hline $60 \mathrm{~min}$ & 477.9 & $37 \cdot 3$ & 329.4 & 5.4 & $405 \cdot 8$ & 4.4 & $135 \cdot 4$ & 3.1 & $135 \cdot 1$ & 4.7 \\
\hline $240 \min$ & 491.4 & 9.4 & 377.5 & 3.0 & $463 \cdot 8$ & $6 \cdot 5$ & $137 \cdot 7$ & 1.4 & $186 \cdot 5$ & $2 \cdot 2$ \\
\hline \multicolumn{11}{|c|}{ Proportion released (\%) } \\
\hline $20 \mathrm{~min} / 60 \mathrm{~min}$ & $86 \cdot 1$ & & $89 \cdot 8$ & & $86 \cdot 8$ & & $91 \cdot 9$ & & $63 \cdot 5$ & \\
\hline $20 \mathrm{~min} / 240 \mathrm{~min}$ & 83.7 & & $78 \cdot 4$ & & $76 \cdot 0$ & & $90 \cdot 3$ & & $46 \cdot 0$ & \\
\hline $60 \mathrm{~min} / 240 \mathrm{~min}$ & $97 \cdot 3$ & & $87 \cdot 3$ & & 87.5 & & $98 \cdot 3$ & & $72 \cdot 4$ & \\
\hline
\end{tabular}

${ }^{*}$ The $\mathrm{GGE}_{20}: \mathrm{GE}_{20}$ ratio shows the effect of allowing for the glycaemic potency of sugars.

$\dagger \mathrm{GGE}=\mathrm{GE}$ adjusted by the glycaemic index of constituent sugars. $\mathrm{GGE}_{20}$ and $\mathrm{GGE}_{60}$ were values used to determine rapidly released GGE and glucose disposal rate, respectively, for a two-point in vitro determination of glycaemic impact.

\section{Statistical analysis}

All data manipulations and statistical analyses were conducted in a Microsoft Excel spreadsheet (Microsoft Corp., Redmond, WA, USA).

\section{Results}

\section{Glucose equivalent determination by in vitro digestion}

The cumulative release of sugars and starch fragments from the foods during digestion, after chewing or homogenising, and measured as glucose equivalents, is shown in Fig. 1 as

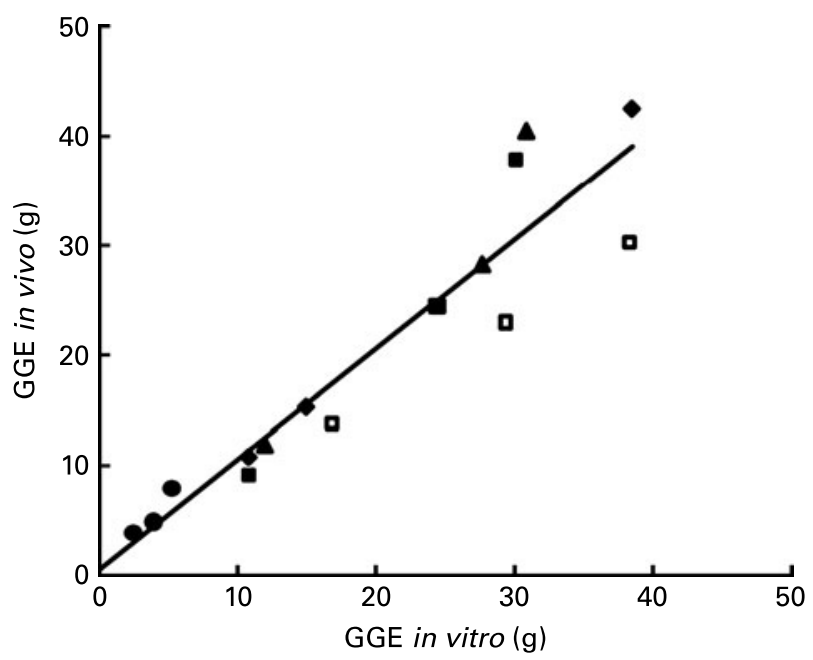

Fig. 7. Correlation between glycaemic glucose equivalent (GGE) content of foods measured as area under the blood glucose response curve (in vivo) $v$. GGE as the area between the lines of GGE release during in vitro digestion and glucose disposal (Fig. 5). ( $\square$ ), Muesli bar; ( $\bullet$ ), potato; ( $\mathbf{\Lambda})$ white bread; $(\bullet)$, chickpeas; $(\boldsymbol{\square})$, fruit loaf; $(-)$, all foods. For white bread, fruit bread, potato, chickpeas, $y=1.156 x-0.54\left(R^{2} 0.96\right)$; for muesli bar, $y=0.761 x+0.99\left(R^{2} 0.99\right)$; for all foods, $y=0.999 x+0.65\left(R^{2} 0.88\right)$. glucose equivalent (mg) per $\mathrm{g}$ of sample. For all foods except the chickpeas most in vitro carbohydrate release had occurred within $20 \mathrm{~min}$ of digestion. For most foods GGE release up to $20 \mathrm{~min}$ of in vitro digestion also accounted for most of the release at $60 \mathrm{~min}$ (white bread $86.1 \%$; fruit bread, $89.8 \%$; muesli bar, $86.8 \%$; potato, $91.9 \%$; chickpeas, $63.5 \%$; Table 3), and at $60 \mathrm{~min}$ most of the total digestible (240 min) GGEs had been released (white bread, 97.3\%; fruit bread, 87.3\%; muesli bar, $87.5 \%$; potato, $98.3 \%$; chickpeas, $72.4 \%$; Table 2).

\section{Glycaemic glucose equivalent determination from glucose equivalents}

GGE values were obtained from glucose equivalent (GE) values by adjusting for the glycaemic potency of the fructose present. The need for the fructose adjustment was confirmed by the improved correlation with in vivo data that the adjustment provided:

$\mathrm{GGE}_{\text {in vivo }}=0 \cdot 557 \mathrm{GE}_{20}$ in vitro $+3 \cdot 016 ; R^{2} 0 \cdot 806$, without the fructose adjustment;

$\mathrm{GGE}_{\text {in vivo }}=0.713 \mathrm{GGE}_{20}$ in vitro $+1.040 ; R^{2} 0.913$, with the adjustment.

\section{Blood glucose responses}

The all-subject mean glucose response curves are shown in Fig. 6(b) (in vivo). Most of the difference in area under the curve for different food intakes could be attributed to changes in the post-peak area. The time to peak did not differ greatly between samples and was unrelated to food intake when all foods were considered (time to peak $\left.(\min )=0.249 \mathrm{GGE}+31 \cdot 31 ; R^{2} 0 \cdot 005\right)$, and the peak heights did not increase linearly with food intake. For most of the blood glucose response curves the area could be divided into pre- and post-peak triangles, which covered most of the area under the curve, and the decrease in blood glucose 
concentrations was almost linear from the peak to baseline, which allowed triangulation to be used to determine a GD rate.

The maximum rate of net glycaemic loading in vivo was between the 15 and $30 \mathrm{~min}$ blood glucose measurements for all intakes, and extrapolating the line from the 15 and 30 min readings back to the $x$-axis showed a delay in response of about $10 \mathrm{~min}$ from consuming food (Fig. 2). Therefore, the curves of GGE release from different food quantities based on in vitro analysis (for example, Fig. 5 for muesli bar) were moved $10 \mathrm{~min}$ along the $x$-axis relative to the original in vitro curves (Fig. 1), so that the start of in vitro carbohydrate release and in vivo blood glucose loading coincided. In vitro GGE release at $20 \mathrm{~min}$ then coincided with the $30 \mathrm{~min}$ upper end of the linear increase in blood glucose, which closely preceded the blood glucose response peak for all foods except chickpeas (Figs. 2 and 6(b)).

\section{Corrections for dose-response non-linearity}

The blood glucose responses to 25,50 and $75 \mathrm{~g}$ glucose, relative to the response to $12.5 \mathrm{~g}$ glucose (assigned a value of 1.0), yielded a quadratic curve, which diverged substantially from a line through zero and 1.0 (Fig. 3). To remove the influence of homeostasis on the glucose reference the blood glucose response curve was multiplied by the ratio of the linear curve (unaffected by homeostasis) to the quadratic curve (resulting from homeostasis).

\section{Glucose disposal rates}

The rate of GD was calculated from a post-peak triangle in which the hypotenuse was a section of the linear slope of decreasing blood glucose concentrations (Fig. 2). The GD rates, calculated as GGE/min and multiplied by the linear: quadratic adjustment in the reference, when plotted against GGE intakes gave a line (Fig. 4) with the equation:

$$
\mathrm{GD} \text { rate }=0.0135 \mathrm{GGE}+0 \cdot 0232(\mathrm{GGE} / \mathrm{min}),
$$

which allowed the GGE disposal rate for a given GGE intake to be calculated. Because the slopes from which the GD rates were calculated all finished between 40 and $90 \mathrm{~min}$, the GGE quantities released at $60 \mathrm{~min}$ in vitro, when most of the digestion of the foods was complete and not very different from the $20 \mathrm{~min}$ value (Table 2), were used in the calculation.

\section{Modelling the interaction of glycaemic loading and glucose disposal}

The in vitro digestion curves for GGE/g sample (Fig. 1 curves of glucose equivalents adjusted to GGE values by allowing for glycaemic potency of the constituent monomers) were multiplied by the food quantities consumed (Table 1), to give curves of theoretical GGE yield per portion consumed. As an example, the curves for muesli bar GGE intakes are shown in Fig. 5 along with a line of cumulative GD for each portion consumed, the latter based on the GD rates appropriate for the GGE intake in each portion consumed (Fig. 4), multiplied by time. The results in Fig. 5 show how the slope of GD increases with the GGE dose consumed. Subtracting values for cumulative GGE disposal at each time from the values for cumulative digestive release of GGE at the same time gave the net GGE loading at the time (Table 2). When these values for net in vitro GGE loading at each time were plotted against time, curves showing how much cumulative GGE loading was exceeding cumulative GGE disposal were obtained that mimicked in vivo blood glucose responses to different foods and food intakes (Fig. 6).

\section{Measuring net glycaemic glucose equivalent content using in vitro area between the curves}

Incremental areas under the in vitro net GGE release curves (incremental area under the curve in vitro $_{1} \mathrm{iAUC}_{\text {in vitro }}$ ) accurately predicted the in vivo responses (Fig. 7) over the range of food intakes (Table 4) $(y=0.999 x+0.65$; $R^{2}$ 0.88). The correlation was much closer if the muesli bar was excluded from the analysis, but for the muesli bar alone the correlation was an almost perfect function of GGE intake, although the slope of the relationship showed that the in vitro analysis gave higher GGE values than the in vivo analysis. A Bland-Altman analysis ${ }^{(13)}$ showed that for the whole dataset the in vitro and in vivo methods corresponded well (Table 4).

Table 4. Relationship between glycaemic glucose equivalents (GGE) determined in vivo* $(y)$ and GGE determined in vitro $(x)$ either from the area between the in vitro GGE release and glucose disposal lines, or from carbohydrate release at $20 \mathrm{~min}$

\begin{tabular}{|c|c|c|c|c|}
\hline \multirow[b]{2}{*}{ Samples } & \multicolumn{2}{|c|}{ Correlation equation } & \multicolumn{2}{|c|}{ Bland-Altman equation } \\
\hline & $y$ & $R^{2}$ & $y$ & $R^{2}$ \\
\hline \multicolumn{5}{|c|}{ GGE measured as area between lines of release and glucose disposal } \\
\hline All foods & $0.999 x+0.65$ & 0.88 & $-0.065 x+0.681$ & 0.032 \\
\hline Muesli bar excluded & $1.103 x-1.532$ & 0.97 & - & - \\
\hline Muesli bar alone & $0.606 x+2 \cdot 843$ & 0.99 & - & - \\
\hline \multicolumn{5}{|c|}{ Two-point analysis based on $\mathrm{GE}_{20}$} \\
\hline $\mathrm{GE}_{20}$ & $0.557 x+3.006$ & 0.81 & $0.491 x-1.89$ & 0.56 \\
\hline $\mathrm{GGE}_{20}$ & $0.713 x+1.040$ & 0.91 & $0.297 x-0.32$ & 0.51 \\
\hline $\mathrm{GGE}_{20}$ - glucose disposal & $1.01 x+2 \cdot 603$ & 0.93 & $-0.048 x-1.86$ & 0.03 \\
\hline
\end{tabular}

$\mathrm{GE}_{20}$, glucose equivalents released after $20 \mathrm{~min}$ in vitro digestion.

${ }^{\star}$ Venn et al. ${ }^{(8)}$. 


\section{Two-point analysis of net glycaemic glucose equivalents}

A two-point analysis of GGE was achieved using the values for GGE release at $20 \mathrm{~min}$, multiplying by food intake, and subtracting the cumulative GD that would have occurred at $20 \mathrm{~min}$.

$$
\text { Net } \mathrm{GGE}_{20}=\mathrm{GGE}_{20}-20\left(0 \cdot 0135 \mathrm{GGE}_{20}+0 \cdot 0232\right)(\mathrm{g}) \text {. }
$$

Correlations of GGE determined by the two-point analysis with GGE in vitro are given in Table 4.

\section{Discussion}

The present study has addressed the challenge of finding a way to express relative glycaemic impact of foods on a grams per consumed quantity of food basis, using a validated in vitro method that mimics the human blood glucose response to foods $^{(1)}$. The ultimate goal is to be able to make relevant comparisons between foods and food intakes in terms of the relative effects of amounts that are customarily consumed. Until this goal is achieved it will be difficult for health professionals, food developers and consumers to make adequate comparisons between alternatives. The principles involved apply generally to the marketing and use of functional foods in promoting health ${ }^{(14)}$.

Our approach in the present study was to combine a highly replicated set of clinically measured responses to multiple intakes of several foods with in vitro analysis in which a number of factors had been addressed to improve physiological relevance:

(1) The samples were chewed before in vitro analysis because of the considerable impact of the physical state of food on digestion ${ }^{(15,16)}$.

(2) The amylolytic phase of digestion was within the gastric-colonic transit time of about $4 \mathrm{~h}$.

(3) The contribution of all carbohydrates to the glycaemic response was taken into account by expressing glycaemic impact in terms of grams of GGE rather than in terms of measured glucose.

(4) Account was taken of the effect of non-linearity of the glucose dose-blood glucose response relationship on the glucose reference values.

(5) Most importantly, in the context of the present paper, baselines were used to represent dose-dependent blood glucose clearance in response to blood glucose loadings from the fifteen food intakes considered.

The model presented here can be regarded as a first iteration and as a test of feasibility in which there were a number of approximations and assumptions, the dataset was small, and the fit between the in vitro (Fig. 6(a)) and in vivo (Fig. 6(b)) response profiles was far from exact. Nonetheless, despite its imperfections, the study has shown that when in vitro digestive analysis uses baselines to represent blood glucose clearance, it is able to mimic in vivo glycaemic responses, and to greatly improve the validity and accuracy with which they are predicted in vitro. The approach has intrinsic validity because it is based on a simulation of the balanced physiological processes known to occur in glucose homeostasis - blood glucose loading and disposal. As key processes in the glycaemic response, both need to be part of any '... validated in vitro methodology that accurately mimics in vivo behaviour' ${ }^{\text {(2) }}$.

Further research is required to address some of the approximations made in the present study. For instance, the GD baselines used in the present model were straight lines, yet insulin release is in response to blood glucose loading, which changes during digestion. Therefore, non-linear GD baselines that track glycaemic loading may give more accurate measures of net effects on blood glucose ${ }^{(17)}$. Also, using the same in vitro digestion curve to create curves for different intakes of a food (Fig. 5) does not allow for possibly more protracted digestion as food intakes increase. The uniformity of the blood glucose response curves (Fig. 6(b)) suggests that this did not greatly influence the results, except perhaps in the case of muesli bars.

The need to build factors into the glycaemic analysis to allow for effects on glycaemic response that are additional to glycaemic carbohydrate intake, such as effects on gastric emptying, should also be considered. A number of studies have suggested that in most foods at normal intakes macronutrient composition has little effect on glycaemic index determination $^{(18,19)}$, and in the present study the relationship between GGE intake and GD for all foods was approximately linear after adjusting for curvature in the glucose reference (Fig. 4). However, others have shown that fat $^{(20)}$, or acid ${ }^{(21)}$, added to a starchy meal causes a large depression of glycaemic response. In the present study the in vitro-in vivo relationship for the muesli bar differed from that of the other foods (Table 4). While the proportional responses to changing intakes of muesli bar were very close for the in vitro and in vivo determinations, the in vitro values were greater than the in vivo. This further suggests that for some food formats additional factors to be included in the analysis of the in vitro results may improve accuracy.

The present paper has shown the importance of taking into account homeostasis and, that by including it in in vitro glycaemic analysis, human blood glucose responses can be mimicked. For routine application, however, a much abbreviated two-point in vitro analysis may be used, in which cumulative GD at $20 \mathrm{~min}$ is subtracted from rapidly released (20 min) GGE, and it provides an almost equally accurate prediction of in vivo responses (JA Monro \& S Mishra, unpublished results). As the maximum glycaemic loading occurred about 20 min after the initiation of the postprandial blood glucose surge, at about $30 \mathrm{~min}$ from food consumption (Figs. 2 and 6(b)), a $20 \mathrm{~min}$ in vitro sampling time appears to be appropriate to measure rapidly available carbohydrate as an indicator of the glycaemic loading responsible for the glycaemic response $\mathrm{e}^{(3,22)}$.

For the experimental approach presented here to be generally applicable as a dose-sensitive in vitro method for predicting the GGE contribution of foods, the relative values that it uses must be generally true. In fact, they are becoming universally established. The relative glycaemic potency of common food mono- and disaccharides required to convert glucose equivalents to GGE values has been measured in numerous studies $^{(11)}$. Similarly, the glucose dose-blood glucose response relationship has been published several times $^{(8,17,18,23-25)}$ and the results, when normalised, show it to be a highly reproducible expression of human physiology ${ }^{(6)}$. 
And assuming that most of the non-linearity in the relationship between glucose dose and glycaemic response is due to increasing GD with increasing glycaemic loading, rates of GD are also likely to be universal when expressed relatively, as in the present study. This view is supported by results from a separate study of the in vitro digestion of eighty-three foods, in which relative GD rates from the present study gave values for net GGE in vitro that predicted GGE in vivo as accurately as in the present study ${ }^{(26)}$.

Because the GD rates, being a function of GGE intake, were based on responses relative to glucose they should be largely immune to effects of individual fluctuations within groups, which introduce enormous variability into clinical determinations of glycaemic response ${ }^{(27,28)}$. Nonetheless, as the range of foods used in the present study was small, and experimental groups are likely to differ in insulin sensitivity, further research is required to firmly establish that relative GD rates are universally applicable and may be used to provide the same GGE values for foods in different population groups. However, if groups that differ in glucose tolerance, such as different age groups, require different GD baselines, such 'customised' baselines could be useful in matching food choices to physiology.

GGE has been defined as a 'virtual food component' because, rather than being a tangible food component per se, it expresses a food effect, but as grams per consumed quantity of food ${ }^{(14)}$. Being in the same weight format as a nutrient, it can easily be used in conjunction with other nutrient data to represent a net glycaemic effect of a whole food, to enable comparisons between foods for glycaemic control. It is, therefore, important that a way is found to allow for the homeostatic modulation of glycaemic responses in response to varying intakes and compositions of foods and meals. The present study has suggested that use of a dose-dependent glucose-disposal factor may overcome the problem, by enabling GGE to be based on a net effect that mimics the human blood glucose response, and is able to be expressed as grams per given quantity of food.

\section{Acknowledgements}

Funding was provided by the New Zealand Foundation for Research, Science and Technology (contract C02X0401).

The contributions of the authors were as follows - J. A. M. conceived of the use of a GD baseline and adjustment for dose-response non-linearity in in vitro glycaemic analysis, planned the experiment, analysed the results, and wrote the paper. S. M. conducted the in vitro analyses and contributed ideas and discussion at all stages. B. V. provided the in vivo glycaemic response curves and led the separate study from which they were obtained ${ }^{(8)}$ with co-authors AJ Wallace, J. A. M., T Perry, R Brown, C Frampton, TJ Green, whose indirect contributions to the present study are gratefully acknowledged.

None of the authors has any personal or financial conflict of interest.

\section{References}

1. Miller-Jones J (2007) Glycemic response definitions. Cereal Foods World 52, 54-55.
2. Brooks S, Craig S \& DeVries J, et al. (2006) Report of the ad hoc Glycemic (Net) Carbohydrate Definition Committee to AACC International Board of Directors. www.aacenet.org/ membership/pdfs/glycemicreport0906.pdf

3. Englyst K, Englyst H, Hudson G, et al. (1999) Rapidly available glucose in foods: an in vitro measurement that reflects the glycemic response. Am J Clin Nutr 69, 448-454.

4. Brighenti F, Pellegrini $\mathrm{N}$, Casiraghi $\mathrm{M}$, et al. (1995) In vitro studies to predict physiological effects of dietary fibre. Eur $J$ Clin Nutr 49, S81-S88.

5. Germaine K, Samman S, Fryirs C, et al. (2008) Comparison of in vitro starch digestibility methods for predicting the glycemic index of grain foods. J Sci Food Agric 88, 652-658.

6. Monro JA \& Shaw M (2008) Glycemic impact, glycemic glucose equivalents, glycemic index, and glycemic load: definitions, distinctions and implications. Am J Clin Nutr 87, 237S-243S.

7. Monro J, Bird A, Topping D, et al. (2009) Database values for food-based control of glycemia. J Food Comp Anal (In the Press).

8. Venn B, Wallace A, Monro J, et al. (2006) Glycemic load estimated from glycaemic index does not differ greatly from glycemic load measured using a standard curve in healthy volunteers. J Nutr 136, 1377-1381.

9. Englyst HN \& Hudson GJ (1987) Colorimetric method for routine analysis of dietary fibre as non-starch polysaccharides. A comparison with gas-liquid chromatography. Food Chem 24, 63-76.

10. Blakeney A \& Mutton L (1980) A simple colourimetric method for determination of sugars in fruit and vegetables. J Sci Food Agric 31, 889-897.

11. Foster-Powell K, Holt SHA \& Brand-Miller JC (2002) International table of glycemic index and glycemic load values: 2002. Am J Clin Nutr 76, 5-56.

12. Food and Agriculture Organization (1998) Carbohydrates in Human Nutrition. Report of a FAO/WHO Expert Consultation on Carbohydrates. FAO Food and Nutrition Paper no. 66. Rome: FAO

13. Bland J \& Altman D (1999) Measuring agreement in method comparison studies. Methods Med Res 8, 135-160.

14. Monro JA (2004) Virtual food components: functional food effects expressed as food components. Eur J Clin Nutr 58, 219-230.

15. Monro J \& Mishra S (2008) Designing digestible carbohydrate particles for controlled energy release. In Cereals 2008, Proceedings of 58th Australian Cereal Chemistry Conference at Gold Coast, pp. 46-49 [J Panozzo and C Black, editors]. North Melbourne, Vic: Royal Australian Chemical Institute.

16. Woolnough J, Monro J, Brennan C, et al. (2008) Simulating human carbohydrate digestion in vitro: a review of methods and the need for standardization. Int J Food Sci Technol 43, 2245-2256.

17. Lee B \& Wolever T (1998) Effect of glucose, sucrose and fructose on plasma glucose and insulin responses in normal humans: comparison with white bread. Eur J Clin Nutr 52, 924-928.

18. Wolever T \& Bolognesi C (1996) Prediction of glucose and insulin responses of normal subjects after consuming mixed meals varying in energy, protein, fat, carbohydrate and glycemic index. J Nutr 126, 2807-2812.

19. Wolever T, Yang M, Zeng X, et al. (2006) Food glycemic index, as given in glycemic index tables, is a significant determinant of glycemic response elicited by composite breakfast meals. Am J Clin Nutr 83, 1306-1312.

20. Henry C, Lightowler H \& Kendall F (2006) The impact of the addition of toppings/fillings on the glycaemic response to commonly consumed carbohydrate foods. Eur J Clin Nutr 60, 763-769. 
21. Liljeberg H \& Bjorck I (1998) Delayed gastric emptying rate may explain improved glycaemia in healthy subjects to a starchy meal with added vinegar. Eur J Clin Nutr 52, 362-371.

22. Mishra S, Monro JA \& Hedderley D (2008) Effect of processing on slowly digestible starch and resistant starch in potatoes. Starch-Stärke 60, 500-507.

23. Jenkins D, Wolever T, Taylor R, et al. (1981) Glycemic index of foods: a physiological basis for carbohydrate exchange. Am J Clin Nutr 34, 362-366.

24. Wolever TMS, Katzmanrelle L, Jenkins AL, et al. (1994) Glycemic index of 102 complex carbohydrate foods in patients with diabetes. Nutr Res 14, 651-669.
25. Wallace A, Monro J, Hedderley D, et al. (2006) Determining the glycemic glucose equivalent value of foods in humans. Nutr Res 26, 47-52.

26. Monro J, Wallace A, Mishra S, et al. (2010) Relative glycaemic impact of customarily consumed portions of 83 foods measured by digesting in vitro and adjusting for food mass and apparent glucose disposal. Br J Nutr (In the Press).

27. Venn B \& Green T (2007) Glycaemic index and glycaemic load: measurement issues and their effect on diet-disease relationships. Eur J Clin Nutr 61, S122-S131.

28. Vega-Lopez S, Griffith J, Ausman L, et al. (2007) Interindividual variability and intraindividual reproducibility of glycemic index values for commercial white bread. Diabetes Care 30, 1412-1417. 